\title{
NMDA receptor genotypes associated with the vulnerability to develop dyskinesia
}

\author{
SA Ivanova', AJM Loonen, ${ }^{2,3}$, P Pechlivanoglou ${ }^{2}$, MB Freidin ${ }^{4,5}$, AFY Al Hadithy ${ }^{2,6}$, EV Rudikov ${ }^{1}$, IA Zhukova ${ }^{7}$, NV Govorin ${ }^{8}$, \\ VA Sorokina ${ }^{9}$, OY Fedorenko ${ }^{1}$, VM Alifirova ${ }^{7}$, AV Semke $^{1}$, JRBJ Brouwers ${ }^{2}$ and B Wilffert ${ }^{2}$
}

Dyskinesias are involuntary muscle movements that occur spontaneously in Huntington's disease (HD) and after long-term treatments for Parkinson's disease (levodopa-induced dyskinesia; LID) or for schizophrenia (tardive dyskinesia, TD). Previous studies suggested that dyskinesias in these three conditions originate from different neuronal pathways that converge on overstimulation of the motor cortex. We hypothesized that the same variants of the $\mathrm{N}$-methyl-D-aspartate receptor gene that were previously associated with the age of dyskinesia onset in HD were also associated with the vulnerability for TD and not LID. Genotyping patients with LID and TD revealed, however, that these two variants were dose-dependently associated with susceptibility to LID, but not TD. This suggested that LID, TD and HD might arise from the same neuronal pathways, but TD results from a different mechanism.

Translational Psychiatry (2012) 2, e67; doi:10.1038/tp.2011.66; published online 10 January 2012

\section{Introduction}

Dyskinesias are involuntary muscle movements that occur as a symptom of Huntington's disease (HD), but also as a complication of long-term treatment with antipsychotic drugs (tardive dyskinesia, TD) or levodopa in Parkinson's disease (on-off phenomena, levodopa-induced dyskinesia, LID). ${ }^{1-4}$ In $\mathrm{HD}$, these involuntary movements have been linked to degeneration of a specific subtype of striatal medium-sized spiny neurons (MSNs) that constitute part of the indirect pathway of the extrapyramidal circuit (Figure 1). ${ }^{5-7}$ Stimulation of these indirect pathway MSN by cortico-striatal glutamatergic neurons results in inhibition of cortical motor areas at the end of this circuit (hypokinesia). ${ }^{8}$ In contrast to dyskinesias in HD, LID is believed to be induced by dopamine D1 receptor bearing direct pathway MSN. ${ }^{4}$ Pulsatile stimulation of these $D 1$ receptors may cause long-term potentiation at $\mathrm{N}$-methyl-D-aspartate (NMDA) receptor of corresponding cortico-striatal synapses, which results in overactivity of the direct pathway MSNs. This leads to activation of cortical areas at the end of this circuit (hyperkinesia). ${ }^{8}$ The mechanism underlying TD is not well understood. This movement disorder occurs after long-term treatment with D2 receptor antagonists and is believed to be caused by damage of the D2 receptor bearing indirect pathway MSNs. It has been hypothesized that this damage is caused by the formation of free radicals induced by long-term treatment. ${ }^{9}$ However, treatment with the antioxidant, vitamin $\mathrm{E}$, did not have convincing effects on TD. ${ }^{9}$ Alternatively, Konitiostis and others reported that NMDA antagonists showed therapeutic effects in an animal model of TD. ${ }^{10}$ Recently, two independent research groups have shown that presence of a specific variant of the NMDA receptor subunit gene is strongly related to the age of onset of dyskinesias in HD. ${ }^{11-13}$ These variants could increase the vulnerability to develop dyskinesia by increasing the sensitivity of indirect pathway MSN to excitotoxicity. As TD is also believed to be related to toxicity at indirect pathway MSN, these variants might also increase the likelihood to develop TD. LID is believed to be related to increased sensitivity of cortico-striatal synapses with direct pathway MSN, and expected to be independent of excitotoxicity at indirect pathway MSN. We therefore hypothesized that these variants might be associated with the likelihood to develop TD and not LID. Our results, to our surprise, are opposite to this hypothesis and make us to question the role of direct pathway MSN in LID.

\section{Subjects and methods}

Patients. In order to test our hypotheses about the role of the abovementioned NMDA receptor variants in TD and LID, we examined a total of 574 Caucasian patients (Supplementary Table 1). Of them, 431 were from three psychiatric hospitals in Tomsk, Kemerovo and Chita oblasts in Siberia. These patients demonstrated schizophrenia $(N=401$, $95.1 \%$ ) or schizotypical disorders according to ICD-10 criteria. All these patients were on long-term treatment with antipsychotic drugs. For comparison, antipsychotic medication doses were converted into chlorpromazine equivalents. ${ }^{14}$ Patients were assessed for the presence or absence of dyskinesia, according to the abnormal involuntary movement

\footnotetext{
${ }^{1}$ Mental Health Research Institute, Tomsk, Russia; ${ }^{2}$ Department of Pharmacy, University of Groningen, Groningen, The Netherlands; ${ }^{3}$ Mental Health Institute Westelijk Noord-Brabant, Halsteren, The Netherlands; ${ }^{4}$ Research Institute for Medical Genetics, Tomsk, Russia; ${ }^{5}$ National Heart and Lung Institute, Imperial College London, London, UK; ${ }^{6}$ Erasmus University Medical Center, Rotterdam, The Netherlands; ${ }^{7}$ Siberian State Medical University, Tomsk, Russia; ${ }^{8}$ Chita State Medical Academy, Chita, Russia and ${ }^{9}$ Kemerovo Regional Clinical Psychiatric Hospital, Kemerovo, Russia

Correspondence: Professor AJM Loonen, Department of Pharmacy, University of Groningen, Antonius Deusinglaan 1, Groningen 9713AV, The Netherlands.

E-mail: a.j.m.loonen@rug.nl

Keywords: extra-pyramidal; GRIN2A; levodopa-induced dyskinesia; medium spiny neuron; NMDA; tardive dyskinesia

Received 1 September 2011; revised 7 November 2011; accepted 6 December 2011
} 


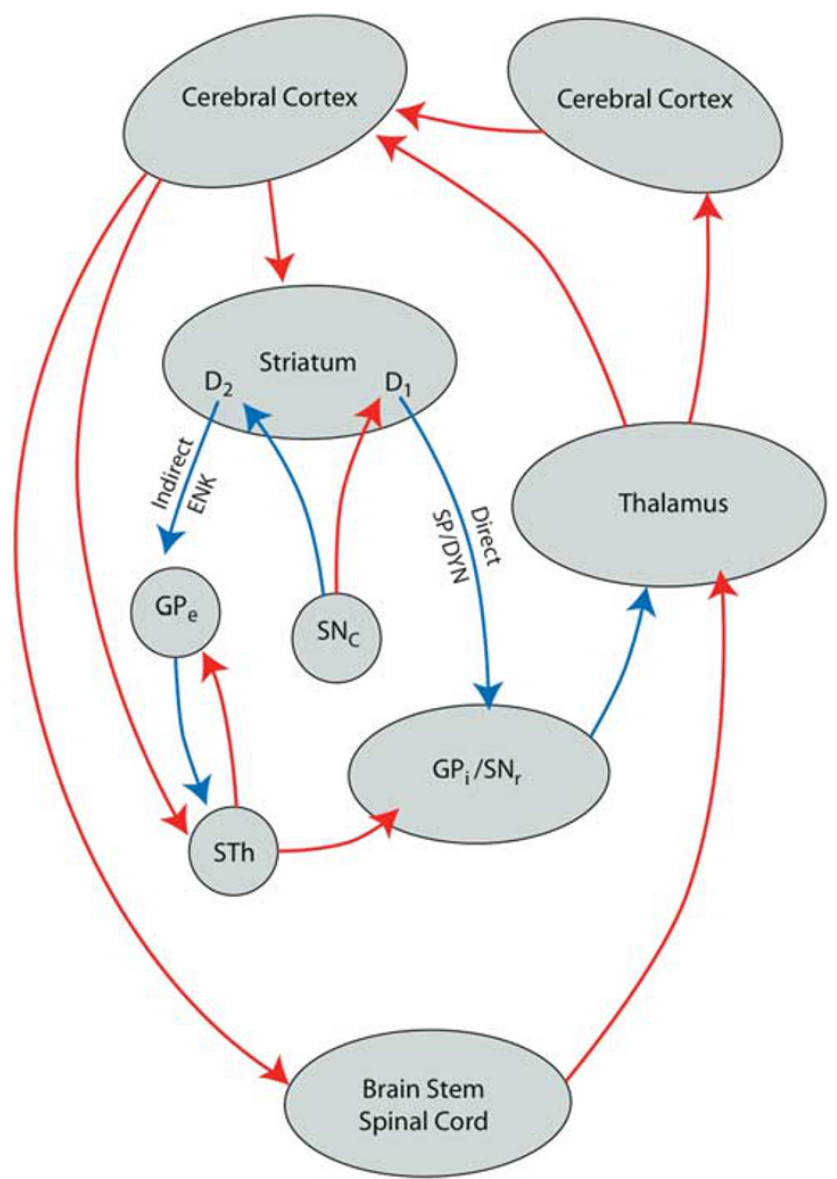

Figure 1 Schematic model of the cortico-striatal-thalamic-cortical (formerly extrapyramidal) circuits, including the indirect and direct pathways; ENK, enkephalin; GPe, Globus pallidus, external segment; GPi, Globus pallidus, internal segment; SNc, Substantia nigra, pars compacta; SNr, substantia nigra, pars reticulata; SP/DYN, substance P/dynorphin; STh, subthalamic nucleus; D1, D2, medium-sized spiny neurons with D1 or D2 receptors.

scale (AIMS) ${ }^{15}$ The AIMS scores were transformed into a binary form (presence or absence of dyskinesia) with Schooler and Kane's criteria. ${ }^{16}$ In addition, a blood sample was taken for DNA isolation and genotyping. We also examined 143 patients with spontaneous extrapyramidal disorders (101 (70.6\%) of them had Parkinson's disease, 21 (14.7\%) had dystonia, 21 had tremor) from the neurology department of the Siberian State Medical University of Tomsk. These patients had not been treated with dopamine antagonists (antipsychotic or antiemetic drugs) for at least 3 years. Of both the groups, other inclusion criteria were no addictions, no current clozapine treatment, no organic disorders and a high quality DNA sample.

DNA analysis. DNA extraction and the Veracode Assay were conducted according to standard protocols. Apart from the to-be studied variants of the genes that encoded two subunits of the glutamatergic NMDA receptor, NR2A and NR2B (GRIN2A and GRIN2B, respectively), we selected a subset of informative SNPs, or tag SNPs, that would accurately represent the majority of SNPs for these genes.
The selection method was previously described by $\mathrm{Xu}$ and Taylor (freely available at http://www.niehs.nih.gov/ snpinfo). ${ }^{17}$ We selected only tag SNPs that captured at least 10 SNPs, including finally 15 SNPs that covered the GRIN2A gene and 9 that covered the GRIN2B gene. For comparison, we also selected a set of variants of 6 unrelated genes that have been reported to be of interest for developing TD. Our final panel consisted of 48 tag SNPs.

Statistical analysis. In order to be more certain about the validity of the results, we used two statistical approaches to analyze the data. Both biostatisticians (MF and PP) independently developed different strategies to account for missing data and interactions between different SNPs. The Hardy-Weinberg equilibrium test was applied to groups with and without dyskinesia. Thereafter, the first biostatistician used the more classical logistic regression and log-linear regression approach. The second biostatistician used the permutation tests and logic regression method. ${ }^{18}$ Because the genotyping for GRIN2A and GRIN2B was definitely hypothesis-driven, no correction for multiple testing was necessary on the primary outcome measure. All calculations were performed in the $R$ statistical environment with the SNPassoc $^{19}$ and LogicReg ${ }^{18}$ package and basic $R$ functions.

\section{Results}

In patients with neurological disorders ( $n=143)$, permutation tests indicated that potential associations existed between LID and the candidate SNPs or combinations of SNPs. In models focused on patients with diagnosed Parkinson's disease $(n=101), 9 \%$ of permutations yielded a deviancebased score smaller than that obtained with the best-fitted model. In models focused on patients with Parkinson's disease that displayed only SNPs of the GRIN2A gene, $4.8 \%$ of permutations yielded a smaller score; this indicated possible associations between the GRIN2A SNPs and LID.

A subsequent Monte Carlo logic regression analysis revealed that two GRIN2A variants, rs7192557 and rs8057394, were most frequently associated with LID observed in all body locations ('all locations'). It is important to notice that rs7192557 is a tagging SNP for rs1969060, which was previously associated with the age of dyskinesia onset in, ${ }^{11,12}$ and that SNP rs8057394 was the other variant associated with $\mathrm{HD} .^{13}$

In an alternative analytical approach, logistic regression was used to test associations between SNPs and dyskinesia. The results indicated that the same two SNPs mentioned above had the highest significance. When the patient sample was limited to patients with diagnosed Parkinson's disease (Supplementary Table 2), a higher odds ratio was observed than that for the entire group of patients with neurological disorders $(n=143)$. For example, the odds ratio for an association between rs7192557 and the 'all locations' LID was 3.21 (95\% Cl: 1.37-7.51, $P=0.0062$ ) for patients diagnosed with Parkinson's disease, and 2.71 (95\% Cl: 1.35-5.46, $P=0.0046)$ for the whole group of patients with neurological disorders. For rs8057394, these figures were $3.59(95 \%$ Cl: $1.48-8.71 ; P=0.0033)$ and $2.86(95 \% \mathrm{Cl}: 1.42-5.76$, 
$P=0.0028)$, respectively. These two SNPs were also significantly $(P<0.05)$ associated with the severity of LID.

Thus, two different statistical methods, the permutation analysis with logic regression and the logistic regression provided similar results.

In the patient population with psychiatric disorders, no relationship could be demonstrated between dyskinesia and the GRIN2A variants, rs7192557 and rs8057394. Moreover, in this patient population, the two different statistical analysis methods produced inconsistent results. Logic regression revealed that the GRIN2B variant rs2192970 and the GRIN2A variant rs1345423 were the most influential of the SNPs tested; however, permutation tests showed almost no association between the SNPs and the outcome. A logistic regression analysis also identified these two SNPs, but only rs1345423 consistently showed highly significant associations with the presence of TD and with the severity of TD.

\section{Discussion}

Our results showed that the susceptibility to LID was associated with two NMDA receptor (GRIN2A) variants, identified by SNPs rs7192557 and rs8057394, which were previously found to be associated with the age of dyskinesia onset in HD. ${ }^{11-13}$ We also found that the susceptibility to TD was associated with two different GRIN2B and GRIN2A variants identified by SNPs rs2192970 and rs1345423, respectively. However, the latter associations were by far less convincing. It should be emphasized that our hypothesis only addressed a possible role for the previously identified SNPs. We selected a panel of 48 SNPs in eight candidate genes. These included 24 SNPs in the genes that encoded GRIN2A and GRIN2B. Therefore, at least some associations should be attributed to chance findings owing to multiple testing. However, when truly associated, the GRIN2A (rs1345423) and GRIN2B (rs2192970) variants are unlikely to be related to the polymorphisms previously found in genetic association studies in schizophrenia ${ }^{20}$ or the GRIN2B variants that were previously studied in TD ${ }^{21}$ and LID. ${ }^{22}$

It should be noticed that limb-truncal and all locations LID show an association with rs7192557 and rs8057394, and that orofacial LID shows no association with any SNP at all. This may correspond with the observation that in HD and LID often large muscle groups are affected and in TD more subtle movements are present, most often in the orofacial area.

An important weakness of our study is the restriction of the patient population to Caucasians from Siberia in Russia. This could also be considered as strength of the study as a more homogenous sample was used. Nevertheless, the need for replication of our findings in an independent patient sample is obvious. In addition, the influence of gender and race should be studied in more detail in a larger patient group. Moreover, the measures to assess TD in general show important limitations. ${ }^{15}$ However, the large number of studied psychiatric patients and our adaptations to the assessment scale give our classification sufficient reliability.

Our results indicate that $H D$ and LID, but not TD, may result from the same biochemical processes involved in the pathology of the extrapyramidal circuit. In HD, this process was linked to NMDA receptor-induced excitotoxicity in the striatal MSNs that form the indirect pathway. ${ }^{5-7}$ Accordingly, this implies that LID may arise from damage to MSNs in the indirect pathway. Our hypothesis is supported by the pathological changes observed in HD, where damage only occurs to MSNs in the indirect pathway.

In contrast, our results showed that TD was not associated with the same NMDA receptor variants. However, antipsychotic drugs are also known to affect MSNs that carry dopamine D2 receptors in the indirect pathway. ${ }^{8}$ Therefore, the MSNs in the indirect pathway may be involved in TD as well, but through a different mechanism.

On the basis of our results for LID, we speculate that the MSNs in the indirect pathway may be particularly susceptible to excitotoxicity during long-term treatment with levodopa owing to the same mechanism that results in progressive degeneration of nigrostriatal dopaminergic neurons in Parkinson's disease. ${ }^{23} \mathrm{~A}$ well-known theory states that Parkinson's disease-induced degeneration is related to the genetically-determined susceptibility of catecholamineexpressing neurons to oxidative stress. ${ }^{23}$ Therefore, we propose that long-term exposure to the catecholamines derived from levodopa may cause the death of MSNs due to a combination of increased susceptibility to oxidative stress and the presence of a variant NMDA receptor that accelerates excitotoxicity.

However, before the above hypothesis can be tested, it is necessary to replicate our finding and to investigate the function of the variant GRIN2A receptor gene in greater detail. It should be established whether the variant NMDA receptor is more active in inducing excitotoxicity and, in addition, whether it is sensitive for NMDA antagonists (for example, memantine or hemantane). It may then be possible to prevent LID by blocking activation of these variant NMDA receptors. Thus, these NMDA antagonists may also be an effective prophylactic for preventing LID in patients that are prone to excitotoxicity during levodopa treatment. Interestingly, the NMDA antagonist amantadine is known to have acute effects on LID, ${ }^{24}$ but memantine lacks this effects. ${ }^{25}$ This can be explained by postulating NMDA antagonism primarily at direct and indirect pathway MSNs, respectively.

\section{Conflict of interest}

The authors declare no conflict of interest.

Acknowledgements. We thank the Genome Analysis Facility ( $\mathrm{Dr} P$ van der Vlies) of the Genetics Department of the University Medical Center, Groningen (UMCG) for their assistance and Dr P Kretchmer, San Francisco Edit, Mill Valley, $\mathrm{CA}$ (www.sfedit.net) for editing the manuscript. We wish to thank Drs Larissa Arning (Ruhr-University, Bochum, Germany), Anton Bespalov (Abbott, Ludwigshafen, Germany), Bernard Lerer (Hebrew University Medical Center, Jerusalem, Israel), and Hans-Jürgen Möller (Ludwig-Maximilians-University, Munich, Germany) for reading the manuscript and their fruitful comments. This research project was supported by the Groningen Center of Drug Research Fund with no external financial support in Russia or the Netherlands.

1. Kane JM. Tardive dyskinesia circa 2006. Am J Psychiatry 2006; 163: 1316-1318.

2. Margolese HC, Chouinard G, Kolivakis TT, Beauclair L, Miller R. Tardive dyskinesia in the era of typical and atypical antipsychotics. part 1: Pathophysiology and mechanisms of induction. Can J Psychiatry 2005; 50: 541-547.

3. Novak MJ, Tabrizi SJ. Huntington's disease. BMJ 2010; 340: c3109.

4. Thanvi B, Lo N, Robinson T. Levodopa-induced dyskinesia in parkinson's disease: Clinical features, pathogenesis, prevention and treatment. Postgrad Med J 2007; 83: 384-388. 
5. Estrada Sanchez AM, Mejia-Toiber J, Massieu L. Excitotoxic neuronal death and the pathogenesis of huntington's disease. Arch Med Res 2008; 39: 265-276.

6. Fan MM, Raymond LA. N-methyl-D-aspartate (NMDA) receptor function and excitotoxicity in huntington's disease. Prog Neurobiol 2007; 81: 272-293.

7. Kumar $\mathrm{P}$, Kalonia H, Kumar A. Huntington's disease: Pathogenesis to animal models Pharmacol Rep 2010; 62: 1-14.

8. Groenewegen HJ. The basal ganglia and motor control. Neural Plast 2003; 10: 107-120

9. Lohr JB, Kuczenski R, Niculescu AB. Oxidative mechanisms and tardive dyskinesia. CNS Drugs 2003; 17: 47-62.

10. Konitsiotis S, Tsironis C, Kiortsis DN, Evangelou A. Effects of N-methyl-D-aspartate receptor antagonism on neuroleptic-induced orofacial dyskinesias. Psychopharmacology (Berl) 2006; 185: 369-377.

11. Arning L, Kraus PH, Valentin S, Saft C, Andrich J, Epplen JT. NR2A and NR2B receptor gene variations modify age at onset in huntington disease. Neurogenetics 2005; 6: 25-28.

12. Andresen JM, Gayan J, Cherny SS, Brocklebank D, Alkorta-Aranburu G, Addis EA et al. Replication of twelve association studies for huntington's disease residual age of onset in large venezuelan kindreds. J Med Genet 2007; 44: 44-50.

13. Arning L, Saft C, Wieczorek S, Andrich J, Kraus PH, Epplen JT. NR2A and NR2B receptor gene variations modify age at onset in huntington disease in a sex-specific manner. Hum Genet 2007; 122: 175-182.

14. Andreasen NC, Pressler M, Nopoulos P, Miller D, Ho BC. Antipsychotic dose equivalents and dose-years: a standardized method for comparing exposure to different drugs. Bio Psychiatry 2010; 67: 255-262.

15. Loonen AJ, van Praag HM. Measuring movement disorders in antipsychotic drug trials: the need to define a new standard. J Clin Psychopharmacol 2007; 27: 423-430.

16. Schooler NR, Kane JM. Research diagnoses for tardive dyskinesia. Arch Gen Psychiatry 1982; 39: 486-487.

17. $\mathrm{Xu} Z$, Taylor JA. SNPinfo: Integrating GWAS and candidate gene information into functional SNP selection for genetic association studies. Nucleic Acids Res 2009; 37: W600-W605.
18. Kooperberg C, Ruczinski I. Identifying interacting SNPs using monte carlo logic regression. Genet Epidemiol 2005; 28: 157-170.

19. Gonzalez JR, Armengol L, Sole X, Guino E, Mercader JM, Estivill X et al. SNPassoc: an $R$ package to perform whole genome association studies. Bioinformatics 2007; 23: 644-645.

20. Cherlyn SY, Woon PS, Liu JJ, Ong WY, Tsai GC, Sim K. Genetic association studies of glutamate, GABA and related genes in schizophrenia and bipolar disorder: A decade of advance. Neurosci Biobehav Rev 2010; 34: 958-977.

21. Liou YJ, Wang YC, Chen JY, Bai YM, Lin CC, Liao DL et al. Association analysis of polymorphisms in the N-methyl-D-aspartate (NMDA) receptor subunit 2B (GRIN2B) gene and tardive dyskinesia in schizophrenia. Psychiatry Res 2007; 153: 271-275.

22. Lee JY, Cho J, Lee EK, Park SS, Jeon BS. Differential genetic susceptibility in diphasic and peak-dose dyskinesias in parkinson's disease. Mov Disord 2011; 26: 73-79.

23. Shadrina MI, Slominsky PA, Limborska SA. Molecular mechanisms of pathogenesis of parkinson's disease. Int Rev Cell Mol Biol 2010; 281: 229-266.

24. Del Dotto P, Pavese N, Gambaccini G, Bernardini S, Metman LV, Chase TN et al. Intravenous amantadine improves levadopa-induced dyskinesias: an acute double-blind placebo-controlled study. Mov Disord 2001; 16: 515-520.

25. Merello M, Nouzeilles MI, Cammarota A, Leiguarda R. Effect of memantine (NMDA antagonist) on parkinson's disease: a double-blind crossover randomized study. Clin Neuropharmacol 1999; 22: 273-276.

Translational Psychiatry is an open-access journal published by Nature Publishing Group. This work is licensed under the Creative Commons Attribution-Noncommercial-Share Alike 3.0 Unported License. To view a copy of this license, visit http:// creativecommons.org/licenses/by-nc-sa/3.0/

\section{Supplementary Information accompanies the paper on the Translational Psychiatry website (http://www.nature.com/tp)}

